\title{
Influence of Visco-Elasticity on MHD Heat and Mass Transfer Flow through a Porous Medium Bounded By an Inclined Surface with Chemical Reaction
}

\author{
Rita Choudhury ${ }^{1^{*}}$ and Bandita Das ${ }^{2}$ \\ Gauhati University, Department of Mathematics, Guwahati, Assam (India), 781014 \\ Email: rchodhury66@yahoo.in
}

\begin{abstract}
The aim of this paper is to investigate the influence of visco-elasticity on steady, two-dimensional flow of an electrically conducting visco-elastic fluid past an inclined surface through a porous medium. The effect of chemical reaction with constant heat and mass flux is also considered into account. The flow is subjected to an external uniform magnetic field normal to the direction of flow. The differential model is termed as second-grade fluid which follows from generalized Rivlin-Ericksen's fluid. Analytical solutions to the coupled non-linear equations governing the flow are obtained by using regular perturbation technique. The expressions for velocity field, temperature field, concentration field, Shearing stress at the plate are derived analytically. The rate of heat transfer and rate of mass transfer in terms of Nusselt number and Sherwood number are also obtained in non-dimensional forms. The results are discussed graphically in possible cases for various combinations of physical parameters. The relevancy of this work has been noticed in the fields of chemical engineering and geophysical applications.
\end{abstract}

Keywords: Visco-elasticity, MHD, Free-convective, Porous medium, Chemical reaction.

\section{INTRODUCTION}

The problem of free convective heat and mass transfer flow of viscous electrically conducting fluid past an inclined surface under the influence of magnetic field has attracted the interest of many researchers in view of its application in aeronautics, fluid fuel nuclear reactor, geophysics, chemical process industries and in many engineering problems. Umemura and Law [1] have studied the natural convection boundary layer flow over a flat plate with arbitrary inclination. They also have noticed that the flow characteristics depend not only on the extent of inclination but also on the distance from the leading edge.Hossain et al. [2] have analyzed the free convective flow from an isothermal plate inclined at small angle to the horizontal. Combined heat and mass transfer flow by free convection past an inclined flat plate has been discussed by Anghel et al. [3]. Chen [4] has analyzed the natural convection flow over a permeable inclined surface with variable wall temperature and concentration. Ganesan and Palani [5] have solved the unsteady natural convection MHD flow past an inclined plate in presence of magnetic field with variable heat and mass flux. Sivasankaran et al. [6] have investigated the Lie group analysis of natural convection heat and mass transfer flow in an inclined surface. Alam and Rahman [7] have developed the MHD free convection and mass transfer flow past an inclined semi-infinite surface in presence of heat generation. Bhuvaneswari et al. [8] have explained exact analysis of radiation convective heat and mass transfer flow over an inclined plate in a porous medium. Kandasamy and Devi [9] have presented the effects of chemical reaction, heat and mass transfer on non-linear laminar boundary-layer flow over a wedge with suction or injection. Prakash and Ogulu [11] have discussed the unsteady two-dimensional flow of a radiating and chemically reacting fluid with time dependent suction. Effects of variable viscosity, heat and mass transfer on nonlinear mixed convection flow over a porous wedge with heat radiation in presence of homogeneous chemical reaction was discussed by kandasamy and Hashim [12]. Kandasamy et al. [13] have explained the thermophoresis and variable viscosity effects on MHD mixed convective heat and mass transfer past a porous wedge in presence of chemical reaction. Jyothi Bala and Vijaya Kumar [14] have studied the unsteady MHD heat and mass transfer flow past a semiinfinite vertical porous moving plate with variable suction in the presence of heat generation and homogeneous chemical reaction. Hitesh Kumar [15] has viewed an analytical solution to the problem of radiative heat and mass transfer over an inclined plate at prescribed heat flux in presence of chemical reaction. The chemical reaction effects on MHD free convective flow through a porous medium bounded by an inclined surface has been analyzed by Mathanrao et al. [16].

The objective of this paper is to study the effects of viscoelasticity on steady two-dimensional free-convection flow of an electrically conducting fluid through porous medium bounded by an inclined surface with constant suction velocity in presence of chemical reaction under the influence of uniform magnetic field applied normal to the direction of 
flow. In the present paper, we have discussed a flow problem associated with second-grade fluid which follows from generalized Rivlin-Ericksen model which encounters both the elasticity and normal stress differences exhibited by most polymeric and biological liquids.

The constitutive equation for the second-order fluid is taken in the form

$$
\sigma=-p I+\mu_{1} A_{1}+\mu_{2} A_{2}+\mu_{3}\left(A_{1}\right)^{2}
$$

where $\sigma$ is the stress tensor, $A_{n}$ are the kinematic RivlinEricksen tensors; $\mu_{1}, \mu_{2}, \mu_{3}$ are material coefficients describing the velocity, elasticity and cross-viscosity respectively. From thermodynamic consideration it is noticed that the material coefficients $\mu_{1}$ and $\mu_{3}$ are positive and $\mu_{2}$ is negative [Coleman and Markovitz [17]]. The equation (1) was derived by Coleman and Noll [18] from that of simple fluids by assuming that stress is more sensitive to the recent deformation than to the deformation that occurred in the distant past.

It is reported that solution of poly-isobutulene in Cetane at $30^{\circ} \mathrm{C}$ simulate a second-order fluid and the material constants for the solutions of various concentrations have been determined by Markovitz.

\section{MATHEMATICAL FORMULATION}

The steady free convective flow of a visco-elastic incompressible electrically conducting fluid through a semiinfinite porous medium bounded by an inclined surface under the influence of uniform magnetic field applied normal to the direction of flow has been studied. The flow is assumed to be in the $x$-direction, which is taken along the semi-infinite inclined plate and y-axis is taken normal to it. The Reynolds number is assumed to be very small and the induced magnetic field due to the flow is neglected. The fluid properties are assumed constant except for the influence of density in the body force term. As the boundary surface is infinite in length, all the variables are functions of $y$ only. Using boundary layer approximation, the governing equations of motion are:

$$
\frac{\partial v}{\partial y}=0
$$

$$
\text { i.e. } v=\text { constant }=-v_{0}
$$

where $v_{0}>0$ corresponding to steady suction velocity at the surface.

$$
\begin{aligned}
& -v_{0} \frac{\partial u}{\partial y}=v_{1} \frac{\partial^{2} u}{\partial y^{2}}+v_{2}\left(-v_{0} \frac{\partial^{3} u}{\partial y^{3}}\right)+g \beta \cos \phi\left(T-T_{\infty}\right)+ \\
& g \beta^{*} \cos \phi\left(C-C_{\infty}\right)-\left(\frac{\sigma B_{0}^{2}}{\rho}\right) \\
& -v_{0} \frac{\partial T}{\partial y}=\frac{\lambda}{\rho C_{p}} \frac{\partial^{2} T}{\partial y^{2}}+\frac{v_{1}}{C_{p}}\left(\frac{\partial u}{\partial y}\right)^{2}+\frac{v_{2}}{C_{p}}\left(-v_{0} \frac{\partial u}{\partial y} \frac{\partial^{2} u}{\partial y^{2}}\right)
\end{aligned}
$$

$$
-v_{0} \frac{\partial C}{\partial y}=D \frac{\partial^{2} C}{\partial y^{2}}+D_{1}\left(C-C_{\infty}\right)
$$

where $u$ and $v$ are the corresponding velocity components along and perpendicular to the surface, $v_{1}$ is viscoelasticity, $g$ is the acceleration due to gravity, $\beta$ is the coefficient of volume expansion for heat transfer and $\beta^{*}$ is the volumetric coefficient of expansion with species concentration, $T$ is the fluid temperature,

$T_{\infty}$ is the temperature of the fluid far away from the plate, $\lambda$ is the thermal conductivity, $\rho$ is the density of the fluid, $C_{p}$ is the specific heat at constant pressure, $C$ is the species concentration, $C_{\infty}$ is the species concentration of the fluid far away from the plate, $D$ is the chemical molecular diffusivity, $D_{1}$ is the rate of chemical reaction and $k$ is the permeability of porous medium, $\varphi$ is the angle of inclination, $\sigma$ is the fluid electrical conductivity, is the magnetic field component along y-axis.

The coresponding boundary conditions of the problem are:

$$
\begin{aligned}
& y=0: u=0, \frac{\partial T}{\partial y}=-\frac{q}{\lambda}, \frac{\partial C}{\partial y}=-\frac{m}{D} \\
& y \rightarrow \infty: u=0, T=T_{\infty}, C=C_{\infty}
\end{aligned}
$$

where $q$ is the heat flux per unit area and $m$ is the mass flux per unit area.

Introducing the non-dimensional parameters

$$
\begin{aligned}
& f(\eta)=\frac{u}{v_{0}}, \eta=\frac{v_{0} y}{v_{1}}, \operatorname{Pr}=\frac{\mu C_{p}}{\lambda}, S c=\frac{v_{1}}{D}, \\
& \theta=\frac{\left(T-T_{\infty}\right) v_{0} \lambda}{q v_{1}}, C^{*}=\frac{\left(C-C_{\infty}\right)}{m v_{1}}, \alpha=\frac{v_{0}^{2} k}{v_{1}}, \\
& G r=\frac{g \beta q v_{1}^{2}}{v_{0}^{4} \lambda}, G m=\frac{g \beta^{*} m v_{1}^{2}}{v_{0}^{4} D}, M=\frac{\sigma B_{0}^{2} v_{1}}{\rho v_{0}^{2}}, \\
& E=\frac{\lambda v_{0}^{2}}{q v_{1} C_{p}}, K r=\frac{v_{1} D_{1}}{v_{0}^{2}}
\end{aligned}
$$

into the equations (3)-(5) and dropping asterisk, we get

$$
\begin{aligned}
& f^{\prime \prime}+f^{\prime}-\alpha_{1} f^{\prime \prime \prime}-\left(M+\frac{1}{\alpha}\right) f=-G r_{1} \theta-G m_{1} C \\
& \theta^{\prime \prime}+\operatorname{Pr} \theta^{\prime}=-E \operatorname{Pr}\left(f^{\prime}\right)^{2}+E \alpha_{1} \operatorname{Pr} f f^{\prime \prime}
\end{aligned}
$$

$C^{\prime \prime}+S c C^{\prime}+K r S c C=0$

The relevant boundary conditions are:

$$
\begin{aligned}
& \eta=0: f=0, \theta^{\prime}=-1, C^{\prime}=-1 \\
& \eta \rightarrow \infty: f \rightarrow \infty, \theta \rightarrow 0, C \rightarrow 0
\end{aligned}
$$

where is the distance, $f(\eta)$ is the velocity, $\operatorname{Pr}$ is the Prandtl number, $S c$ is the Schmidt number, $G r$ is the Grashof number, $G m$ is the solutal Grashof number, $E$ is the Eckert number, $M$ is the magnetic field parameter, $K r$ is the 
chemical reaction parameter, $k$ is permeability of porous medium, $\alpha$ is the permeability parameter, is the elasticoviscous parameter, $\theta$ is the dimensionless temperature, $C$ is the dimensionless concentration, $G r_{1}=G r \cos \varphi$ and $G m_{1}=G m \cos \varphi$ and prime denotes differentiation with respect to.

\section{METHOD OF SOLUTION}

To solve the equations (8) to (10) let us assume

$$
\begin{aligned}
& f(\eta)=f_{0}(\eta)+E f_{1}(\eta)+0\left(E^{2}\right) \\
& \theta(\eta)=\theta_{0}(\eta)+E \theta_{1}(\eta)+0\left(E^{2}\right) \\
& C(\eta)=C_{0}(\eta)+E C_{1}(\eta)+0\left(E^{2}\right)
\end{aligned}
$$

where $E$ is considered as the perturbation parameter.

Substituting (12) into equations (8)-(10) and equating harmonic and non-harmonic terms, and neglecting higher orders of $0\left(E^{2}\right)$, we obtain

$$
\begin{gathered}
f_{0}^{\prime \prime}+f_{0}^{\prime}-\alpha_{1} f_{0}^{\prime \prime \prime}-\left(M+\frac{1}{\alpha}\right) f_{0}=-G r_{1} \theta_{0}-G m_{1} C_{0} \\
f_{1}^{\prime \prime}+f_{1}^{\prime}-\alpha_{1} f_{1}^{\prime \prime \prime}-\left(M+\frac{1}{\alpha}\right) f_{1}=-G r_{1} \theta_{1}-G m_{1} C_{1}
\end{gathered}
$$

$\theta_{0}^{\prime \prime}+\operatorname{Pr} \theta_{0}^{\prime}=0$

$\theta_{1}^{\prime \prime}+\operatorname{Pr} \theta_{1}^{\prime}=-\operatorname{Pr} f_{0}^{2}+\alpha_{1} \operatorname{Pr} f_{0}^{\prime} f_{0}^{\prime \prime}$

$C_{0}^{\prime \prime}+S c C_{0}^{\prime}+K r S c C_{0}=0$

$C_{1}^{\prime \prime}+S c C_{1}^{\prime}+K r S c C_{1}=0$

subject to the boundary conditions:

$$
\begin{aligned}
& \eta=0: f_{0}=0, f_{1}=0, \theta_{0}^{\prime}=-1, \theta_{1}^{\prime}=0, C_{0}^{\prime}=-1, C_{1}^{\prime}=0 \\
& \eta \rightarrow \infty: f_{0} \rightarrow 0, f_{1} \rightarrow 0, \theta_{0} \rightarrow 0, \theta_{1} \rightarrow 0, C_{0} \rightarrow 0, C_{1} \rightarrow 0
\end{aligned}
$$

The solutions of the equations (17) and (18) subject to the boundary conditions (19) are given by

$C_{0}=K_{1} e^{-t_{1} \eta}, C_{1}=0$

To solve the equations (13)-(16), we use multiparameter perturbation scheme following Nowinski and Ismail (19)

(as $\alpha_{1}<<1$ for small rate of shear) as

$$
\begin{aligned}
& f_{0}=f_{00}+\alpha_{1} f_{01}+0\left(\alpha_{1}^{2}\right) \\
& f_{1}=f_{10}+\alpha_{1} f_{11}+0\left(\alpha_{1}^{2}\right) \\
& \theta_{0}=\theta_{00}+\alpha_{1} \theta_{01}+0\left(\alpha_{1}^{2}\right) \\
& \theta_{1}=\theta_{10}+\alpha_{1} \theta_{11}+0\left(\alpha_{1}^{2}\right)
\end{aligned}
$$

Using (21) into equations (13)-(16) and equating like terms of $\alpha_{1}$ and neglecting those of $\alpha_{1}^{2}$, we get the following differential equations:

\section{Zeroth-order equations:}

$f_{00}^{\prime \prime}+f_{00}^{\prime}-\left(M+\frac{1}{\alpha}\right) f_{00}=-G r_{1} \theta_{00}-G m_{1} C_{0}$

$f_{10}^{\prime \prime}+f_{10}^{\prime}-\left(M+\frac{1}{\alpha}\right) f_{10}=-G r_{1} \theta_{00}-G m_{1} C_{1}$

$\theta_{00}^{\prime \prime}+\operatorname{Pr} \theta_{00}^{\prime}=0$

$\theta_{10}^{\prime \prime}+\operatorname{Pr} \theta_{10}^{\prime}=-\operatorname{Pr}\left(f_{00}^{\prime}\right)^{2}$

\section{First-order equations:}

$f_{01}^{\prime \prime}+f_{01}^{\prime}-f_{00}^{\prime \prime \prime}-\left(M+\frac{1}{\alpha}\right) f_{01}=-G r_{1} \theta_{01}$

$f_{11}^{\prime \prime}+f_{11}^{\prime}-f_{10}^{\prime \prime \prime}-\left(M+\frac{1}{\alpha}\right) f_{11}=-G r_{1} \theta_{11}$

$\theta_{01}^{\prime \prime}+\operatorname{Pr} \theta_{01}^{\prime}=0$

$\theta_{11}^{\prime \prime}+\operatorname{Pr} \theta_{11}^{\prime}=-2 \operatorname{Pr} f_{00}^{\prime} f_{01}^{\prime}+\alpha_{1} \operatorname{Pr} f_{00}^{\prime} f_{00}^{\prime \prime}$

The relevant boundary conditions are

$\eta=0: f_{00}=0, f_{01}=0, f_{10}=0, f_{11}=0$,

$\theta_{00}^{\prime}=-1, \theta_{01}^{\prime}=0, \theta_{10}^{\prime}=0, \theta_{11}^{\prime}=0$

$\eta \rightarrow \infty: f_{00} \rightarrow 0, f_{01} \rightarrow 0, f_{10} \rightarrow 0$,

$f_{11} \rightarrow 0, \theta_{00} \rightarrow 0, \theta_{01} \rightarrow 0, \theta_{10} \rightarrow 0, \theta_{11} \rightarrow 0$

The solutions of the equations (22) to (29) under the boundary conditions (30) are presented as follows:

$$
\begin{aligned}
& f_{00}=K_{2} e^{-t_{2} \eta}-A_{1} e^{-\operatorname{Pr} \eta}-A_{2} e^{-t_{1} \eta} \\
& f_{01}=A_{6} e^{-t_{2} \eta}+A_{5} \eta e^{-t_{2} \eta}+A_{3} e^{-\operatorname{Pr} \eta}+A_{4} e^{-t_{1} \eta} \\
& f_{10}=A_{22} e^{-t_{2} \eta}+A_{14} e^{-\operatorname{Pr} \eta}+A_{15} e^{-2 t_{2} \eta}+A_{16} e^{-2 \operatorname{Pr} \eta} \\
& +A_{17} e^{-2 t_{1} \eta}+A_{18} e^{-\left(t_{2}+\operatorname{Pr}\right) \eta}+A_{19} e^{-\left(t_{1}+\operatorname{Pr}\right) \eta}+A_{20} e^{-\left(t_{2}+t_{1}\right) \eta} \\
& +A_{21} \eta e^{-2 t_{1} \eta} \\
& f_{11}=A_{44} e^{-t_{2} \eta}+A_{34} e^{-\operatorname{Pr} \eta}-A_{35} \eta e^{-2 t_{2} \eta}+A_{36} \eta^{2} e^{-t_{2} \eta} \\
& +A_{37} e^{-\left(t_{2}+\operatorname{Pr}\right) \eta}+A_{38} \eta e^{-\left(t_{1}+\operatorname{Pr}\right) \eta}+A_{39} e^{-\left(t_{2}+t_{1}\right) \eta} \\
& +A_{40} e^{-2 \operatorname{Pr} \eta}+A_{41} e^{-2 t_{2} \eta}+A_{42} e^{-2 t_{1} \eta}+A_{43} \eta e^{-\left(t_{2}+t_{1}\right) \eta} \\
& \theta_{00}=\frac{1}{\operatorname{Pr}} e^{-\operatorname{Pr} \eta}, \\
& \theta_{01}=0 \\
& \theta_{10}=A_{13} e^{-\operatorname{Pr} \eta}+A_{7} e^{-2 t_{2} \eta}+A_{8} e^{-2 \operatorname{Pr} \eta}+A_{9} \eta e^{-2 t_{1} \eta} \\
& +A_{10} e^{-\left(t_{2}+\operatorname{Pr}\right) \eta}+A_{11} e^{-\left(t_{1}+\operatorname{Pr}\right) \eta}+A_{12} e^{-\left(t_{2}+t_{1}\right) \eta}
\end{aligned}
$$


$\theta_{11}=A_{23} e^{-t_{2} \eta}+A_{24} \eta e^{-t_{2} \eta}$

$+A_{25} e^{-\left(t_{2}+\mathrm{Pr}\right) \eta}+A_{26} \eta e^{-\left(t_{2}+\mathrm{Pr}\right) \eta}+A_{27} e^{-\left(t_{2}+t_{1}\right) \eta}+$

$A_{28} \eta e^{-\left(t_{2}+t_{1}\right) \eta}+A_{29} e^{-\left(t_{1}+\operatorname{Pr}\right) \eta}-A_{30} \eta e^{-2 t_{1} \eta}+$

$A_{31} e^{-2 \operatorname{Pr} \eta}+A_{32} e^{-\operatorname{Pr} \eta}$ by

The fluid velocity, temperature and concentration are given

$f=\left(f_{00}+\alpha_{1} f_{01}\right)+E\left(f_{10}+\alpha_{1} f_{11}\right)$

$\theta=\left(\theta_{00}+\alpha_{1} \theta_{01}\right)+E\left(\theta_{10}+\alpha_{1} \theta_{11}\right)$

$C=C_{0}+E C_{1}$

The non-dimensional form of shearing stress coefficient at the surface is given by

$\sigma=\left(\frac{\sigma_{x y}}{\rho v_{0}^{2}}\right)_{y=0}=\left(\frac{\partial f}{\partial \eta}\right)_{\eta=0}=f^{\prime}(0)+\alpha_{1} f^{\prime \prime}(0)$

The non-dimensional heat flux in terms of Nusselt number is given by

$N u=\left(\frac{\partial \theta}{\partial \eta}\right)_{\eta=0}=-1+A_{45}$

The mass flux in terms of Sherwood number is given by

$S h=\left(\frac{\partial C}{\partial \eta}\right)_{\eta=0}=A_{46}$

where the constants are obtained but not presented here due to sake of brevity.

\section{RESULTS AND DISCUSSION}

The steady two-dimensional MHD free convective viscoelastic flow through a porous medium bounded by inclined surface in presence of chemical reaction with heat and mass transfer has been formulated, analyzed and solved by using multi parameter perturbation scheme. Approximate solutions have been derived for velocity, temperature, concentration and shearing stress. The non-dimensional parameter $\alpha_{1}=0$ characterizes the Newtonian fluid phenomenon and non-zero values of $\alpha_{1}$ exhibit the visco-elastic fluid.

Figures 1-7 depict the fluid velocity $f(\eta)$ against $\eta$ for several values of Prandtl number (Pr), Schmidt number (Sc), Grashof number for heat transfer (Gr), Grashof number for mass transfer $(\mathrm{Gm})$,Eckert number $(\mathrm{E})$, permeability parameter $(\mathrm{k})$, chemical reaction parameter $(\mathrm{Kr})$ and viscoelastic parameter $\left(\alpha_{1}\right)$,with fixed values of $\varepsilon=0.3, \alpha==1$.

\section{FIGURES}

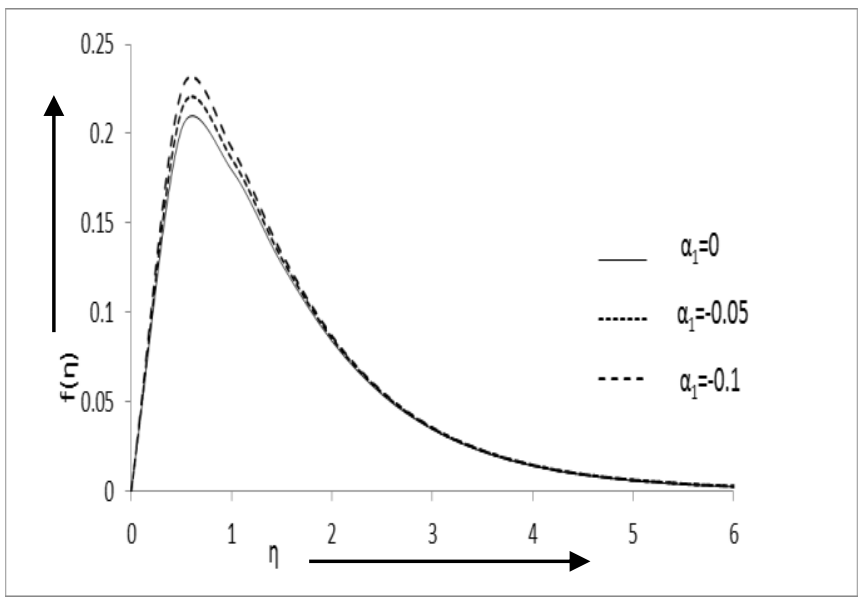

Figure 1. Fluid velocity $f(\eta)$ against $\eta$ for $\operatorname{Pr}=3, S c=1, G m=2$, $\mathrm{Gr}=1, \mathrm{Kr}=0.1, \mathrm{M}=3$

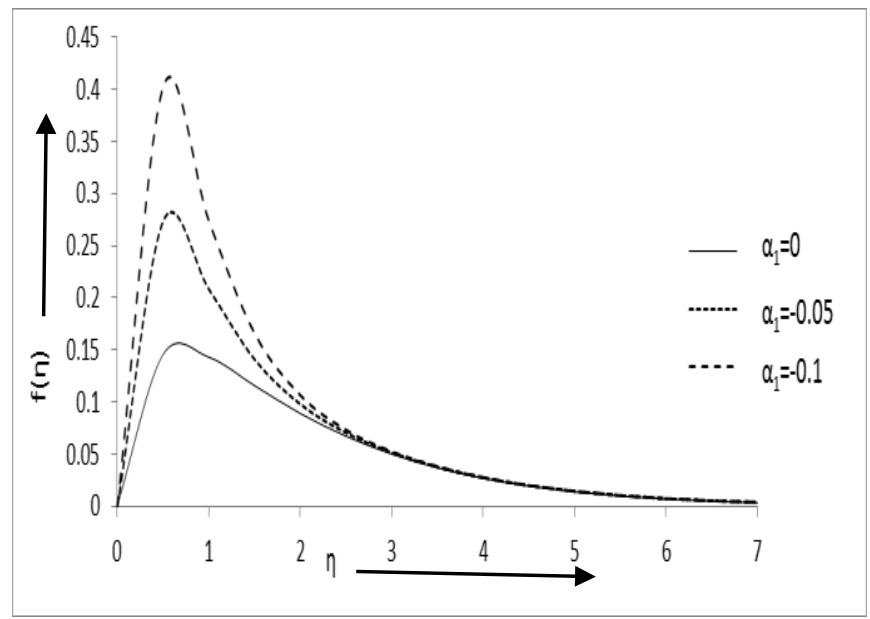

Figure 2. Fluid velocity $f(\eta)$ against $\eta$ for $\operatorname{Pr}=3, S c=1, G m=2$, $\mathrm{Gr}=1, \mathrm{Kr}=0.2, \mathrm{M}=3$

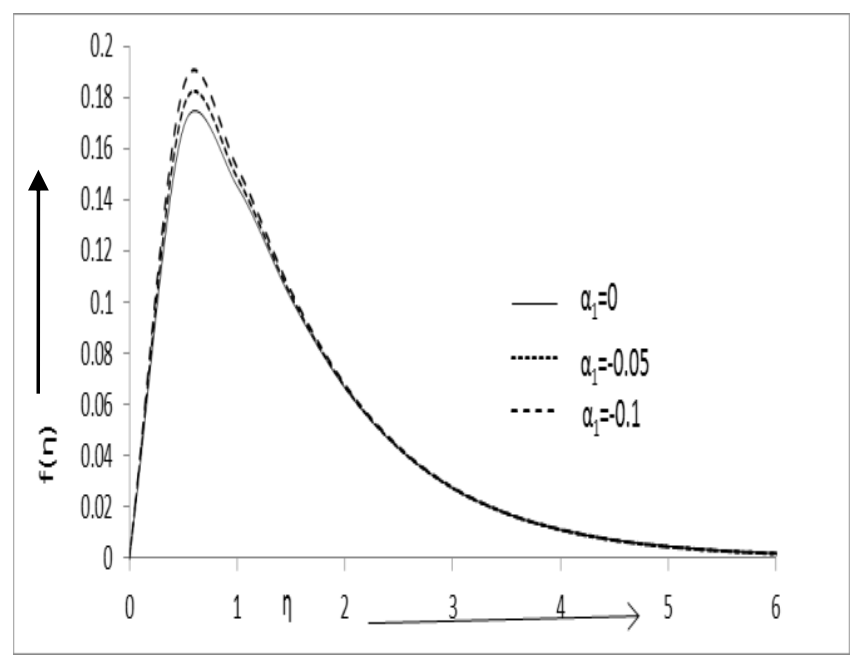

Figure 3. Fluid velocity $f(\eta)$ against $\eta$ for $\operatorname{Pr}=3, S c=1, G m=2$, $\mathrm{Gr}=1, \mathrm{Kr}=0.1, \mathrm{M}=4$ 


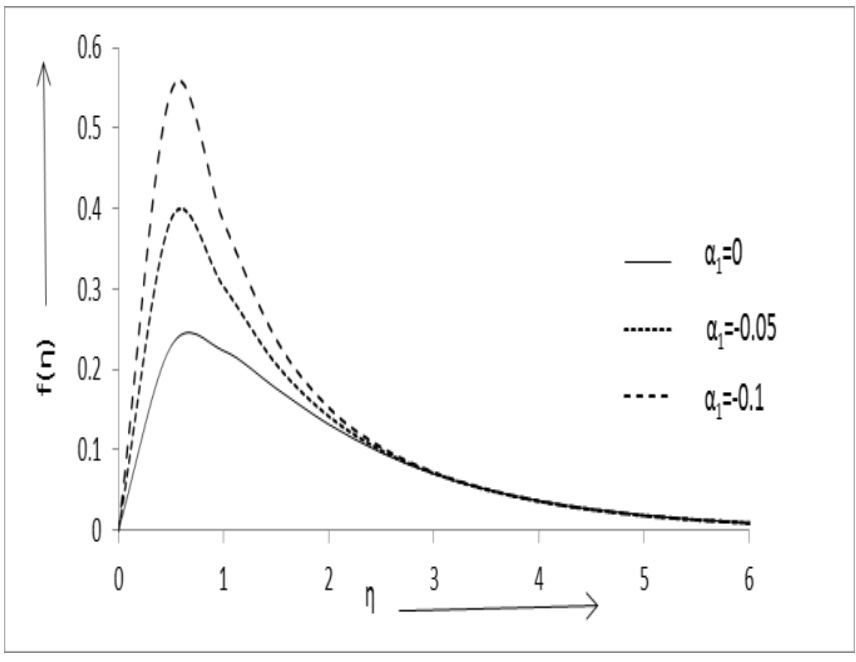

Figure 4. Fluid velocity $f(\eta)$ against $\eta$ for $\operatorname{Pr}=3.5, \mathrm{Sc}=0.8$, $\mathrm{Gm}=2, \mathrm{Gr}=1, \mathrm{Kr}=0.1, \mathrm{M}=3$

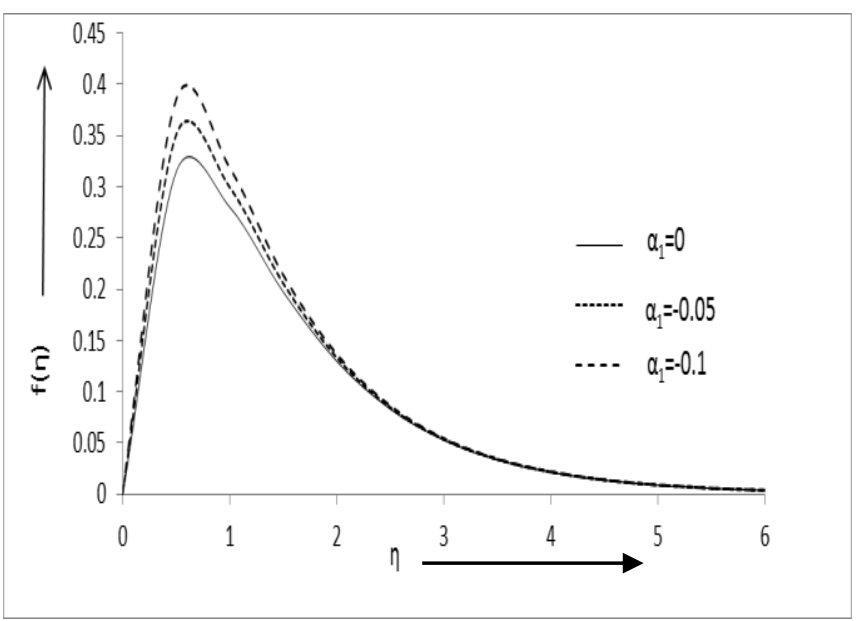

Figure 5. Fluid velocity $f(\eta)$ against $\eta$ for $\operatorname{Pr}=3, S c=1, G m=3$, $\mathrm{Gr}=1, \mathrm{Kr}=0.1, \mathrm{M}=3$

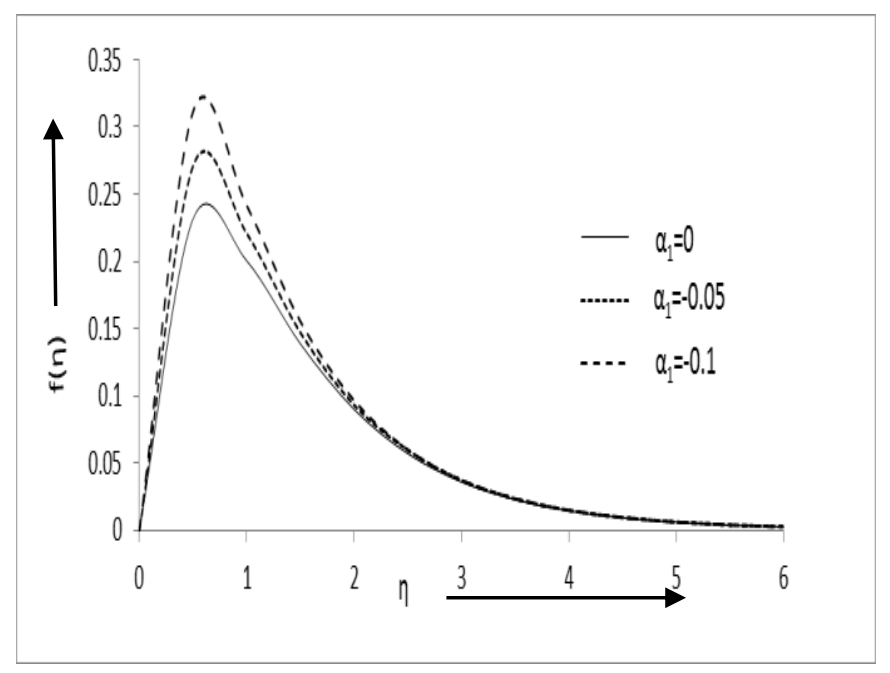

Figure 6. Fluid velocity $f(\eta)$ against $\eta$ for $\operatorname{Pr}=3, S c=1, G m=2$, $\mathrm{Gr}=2, \mathrm{Kr}=0.1, \mathrm{M}=3$

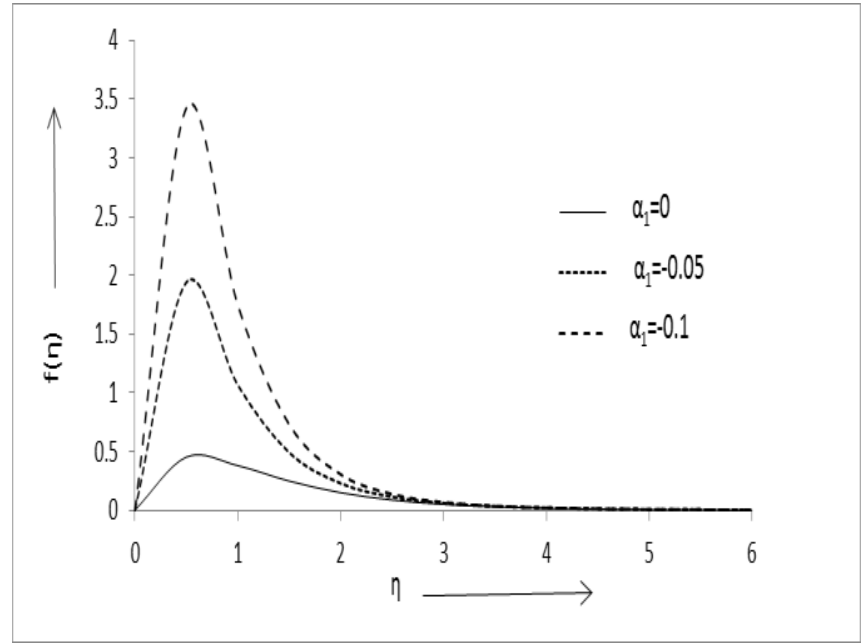

Figure 7. Fluid velocity $f(\eta)$ against $\eta$ for $\operatorname{Pr}=3.5, S c=1$, $\mathrm{Gm}=2, \mathrm{Gr}=1, \mathrm{Kr}=0.1, \mathrm{M}=3$

It is evident from the figures 1-7 that the fluid velocity $f(\eta)$ accelerate near the plate and uniformly away from the plate in both Newtonian and non-Newtonian cases. The rising trend of absolute value of visco-elastic parameter $\left|\alpha_{1}\right|\left(\alpha_{1}=0,-0.05,-0.1\right)$ |increases the fluid velocity in comparison with Newtonian fluid.

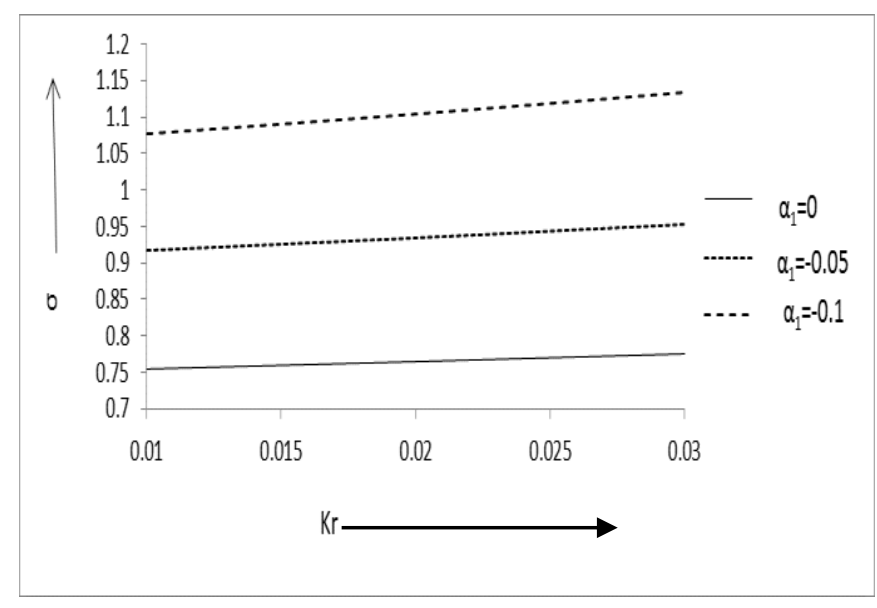

Figure 8. Shearing stress $\sigma$ against Chemical reaction parameter $(\mathrm{Kr})$ for $\mathrm{Pr}=3, \mathrm{Sc}=1, \mathrm{Gm}=2, \mathrm{Gr}=1, \mathrm{M}=3$

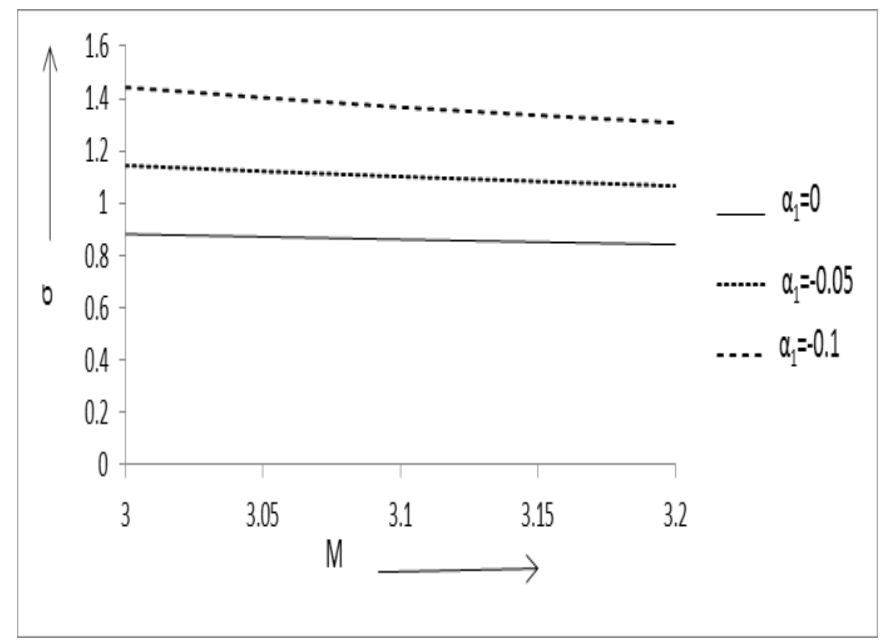

Figure 9. Shearing stress $\sigma$ against magnetic parameter (M) for $\mathrm{Pr}=3, \mathrm{Sc}=1, \mathrm{Gm}=2, \mathrm{Gr}=1, \mathrm{Kr}=0.1$ 


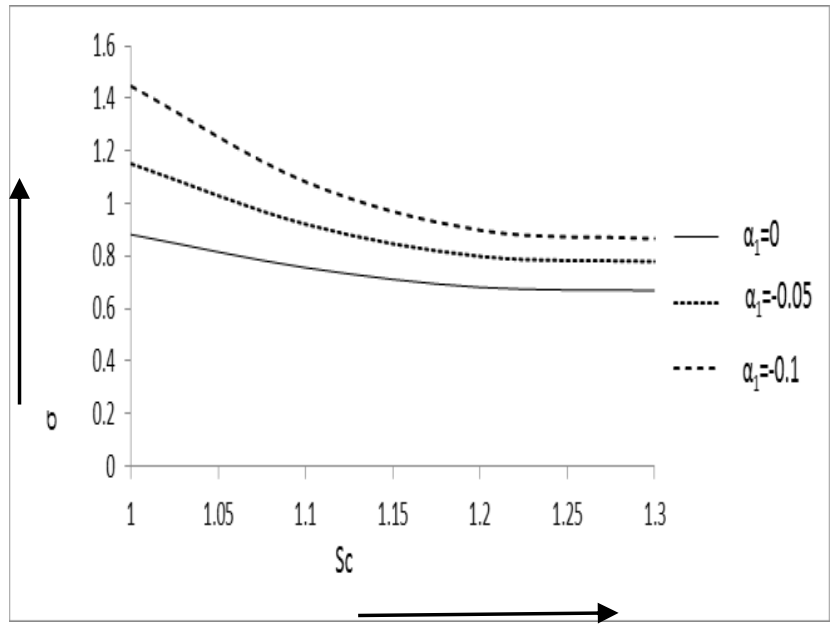

Figure 10. Shearing stress $\sigma$ against $\mathrm{Schmidt}$ number $(\mathrm{Sc})$ for $\mathrm{Pr}=3, \mathrm{Gr}=5, \mathrm{Gm}=5, \mathrm{Kr}=0.1, \mathrm{M}=3$

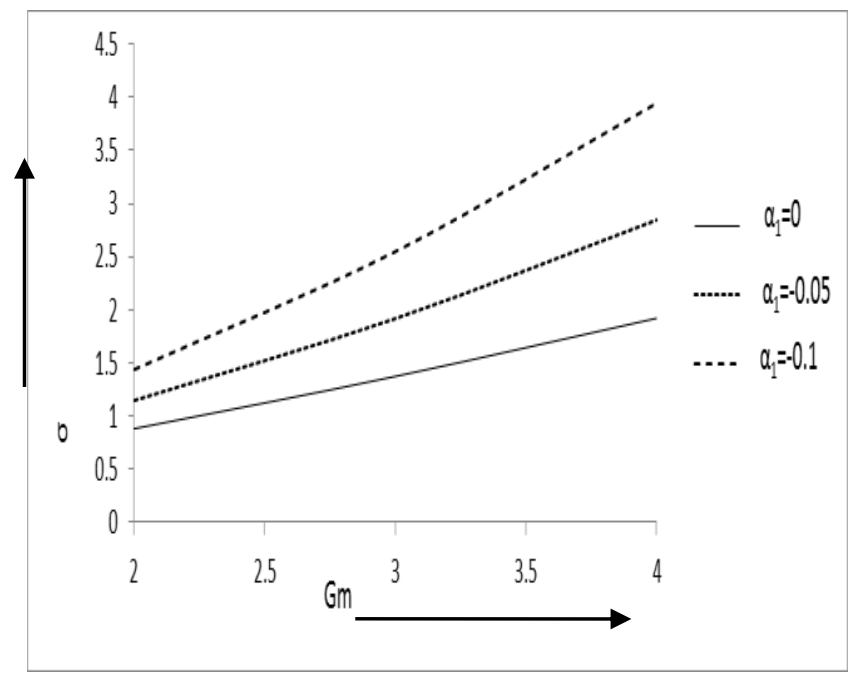

Figure 11. Shearing stress $\sigma$ against solutal Grash of number $(\mathrm{Gm})$ for $\mathrm{Pr}=3, \mathrm{Sc}=1, \mathrm{Gr}=1, \mathrm{Kr}=0.1, \mathrm{M}=3$

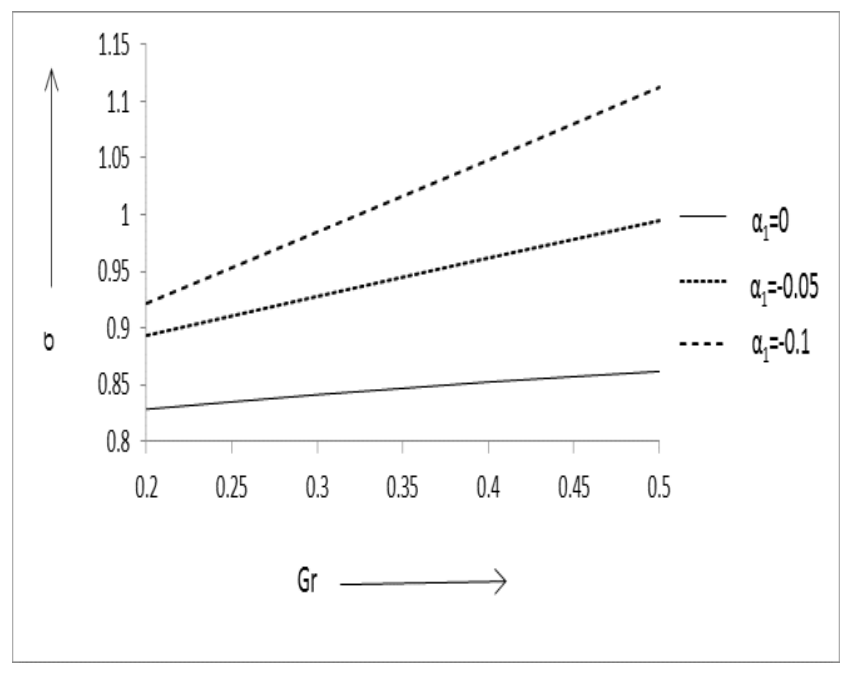

Figure 12. Shearing stress $\sigma$ against Grashof number for heat transfer $(\mathrm{Gr})$ for $\mathrm{Pr}=3, \mathrm{Sc}=1, \mathrm{Gm}=2, \mathrm{Kr}=0.1, \mathrm{M}=3$

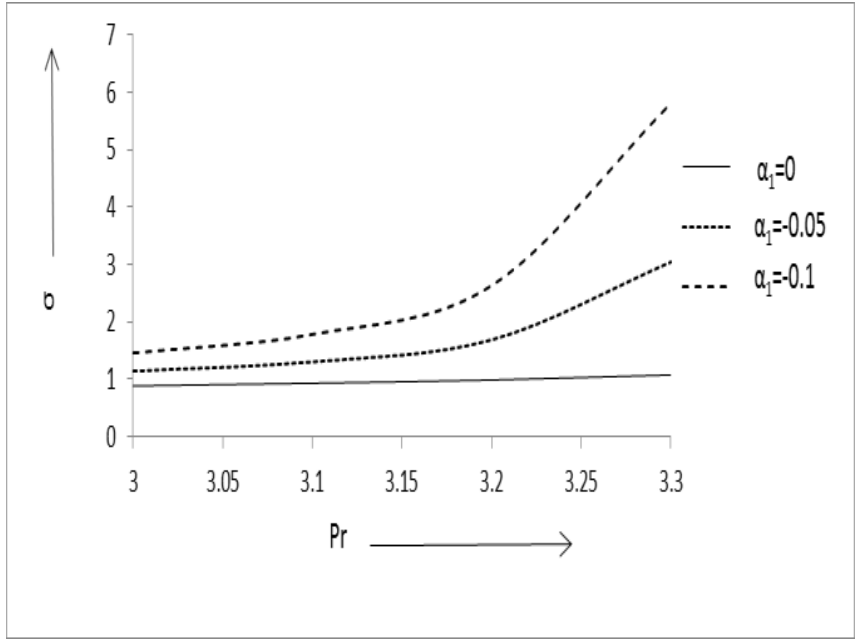

Figure 13. Shearing stress $\sigma$ against Prandtl number $(\mathrm{Pr})$ for $\mathrm{Sc}=1, \mathrm{Gm}=2, \mathrm{Gr}=1, \mathrm{Kr}=0.1, \mathrm{M}=3$

The figures 8-13 exhibit the effects of the visco-elastic parameter $\left|\alpha_{1}\right|$ on shearing stress $\sigma$ against Chemical reaction parameter Kr, Magnetic parameter M,Schmidt number Sc, solutal Grashof number Gm, Grashof number for heat transfer $\mathrm{Gr}$ and Prandtl number Pr with the fixed values of $\alpha=1, \varepsilon=0.3$ to observe the visco-elastic effects. The figures reveal that the shearing stress coefficient $\sigma$ increases with the increasing values of visco-elastic parameter $\left|\alpha_{1}\right|$ compared to Newtonian fluid. It is also noted that shearing stress increases with the increase of Chemical reaction parameter, solutal Grashof number Gm, Grashof number for heat transfer $\mathrm{Gr}$, and Prandtl number Pr but reverse trend is observed in case of Schmidt number Sc and magnetic parameter M.

It has also been observed that the temperature field, concentration field, Nusselt number and Sherwood number are not significantly affected by the visco-elastic parameter.

\section{CONCLUSION}

The present study gives an analytical treatment about the effects of visco-elasticity on the steady MHD flow with heat and mass transfer in presence of chemical reaction. From the results the following conclusions could be drawn:

(l) The flow field is considerably affected by the viscoelastic parameter.

(2) The influence of visco-elasticity is maximum near the surface of the wall in combination of other physical parameters.

(3) The velocity of the fluid flow is affected by the magnetic parameter. It is seen that with the enhancement of magnetic parameter the velocity diminishes and this is matched with physical behavior of magnetic parameter.

(4) The rate of heat transfer and rate of mass transfer are not significantly affected by the visco-elastic parameter in the fluid flow region.

\section{REFERENCES}

[1] A. Umemura and C. K. Law, "Natural convection boundary layer flow over a heated plate with arbitrary inclination," J. Fluid Mech., vol. 219, pp. 571-584, 1990. DOI: 10. 1017/S0022112090003081.

[2] M. A. Hossain, I. Pop and M. Ahmad, "MHD free 
convection flow from an isothermal plate inclined at a small angle to the horizontal," J. Theo. Appl. Fluid Mech., vol. 1, pp. 194-207, 1996.

[3] M. Anghel. M. A. Hossain, S. Zeb. and I. Pop. I, "Combined heat and mass transfer by free convection past an inclined flate plate," Int. J. Appl. Mech. and Engng, vol. 2, pp. 219-235, 2001.

[4] C. H. Chen, "Heat and mass transfer in MHD flow by natural convection from a permeable inclined surface with variable wall temperature and concentration," Acta Mechanica., vol. 172, pp. 219-235, 2004. DOI: 10. 1007/S00707-004-0155-5.

[5] P. Ganesan and G. Palani, "Finite difference analysis of unsteady natural convection MHD flow past an inclined plate with variable heat and mass flux," Int. J. Heat Mass transfer., vol. 47, pp. 4449-4457, 2004. DOI: $10.1016 /$ j. ijheat mass transfer 2004. 04. 034.

[6] S. Sivasankaran, M. Bhuvaneswari, P. Kandaswamy, and E. K. Ramasami, "Lie group analysis of natural convection heat and mass transfer in an inclined surface, non-linear analysis"; Modelin and Control., vol. II. No. 1, pp. 201-212, 2006.

[7] M. S. Alam and M. M Rahman, "MHD free convective heat and mass transfer flow past an inclined surface with heat generation," Thammasat Int. J. Sc. Tech., vol. II, No. 4, pp. 1-8, 2006.

[8] M. Bhuvaneswari, S. Sivasankaran and Y. J. Kim, "Exact analysis of radiation convective flow heat and mass transfer over an inclined plate in a porous medium," World Applied Sciences Journal, vol. 10, pp. 774-778, 2010.

[9] R. Kandasamy and Anjali Devi, S. P., "Effects of chemical reaction, heat and mass transfer on non-linear laminar boundary -layer flow over a wedge with suction or injection," Journal of computational and Applied Mechanics, vol. 5, pp. 21-31, 2004.

[10] R. Kandasamy, K. Periasamy and K. K. Prabhu Sivagnana.,"Effects of chemical reaction, heat and mass transfer along a wedge with heat source and concentration in presence of suction or injection," Int. J. Heat Mass Transfer, vol. 48, pp. 1388-1396, 2005.

[11] J. Prakash and A. Ogulu., "Unsteady two-dimensional flow of a radiating and chemically reacting MHD fluid with time dependent suction," Ind. J. Pure \& Appl. Phys., vol. 44, pp. 805-810, 2006.

[12] R. Kandasamy, I. Hashim, "Effects of variable viscosity, heat and mass transfer on nonlinear mixed convection flow over a porous wedge with heat radiation in the presence of homogeneous chemical reaction," ARPN. J. Engg. Applied Sci., vol. 2, pp. 4453, 2009.

[13] R. Kandasamy, I. Muhaimin and Khamis, B. Azme, "Thermophoresis and variable viscosity effects on MHD mixed convective heat and mass transfer past a porous wedge in the presence chemical reaction, Heat and Mass transfer past a porous wedge in the presence of chemical reaction," Heat and Mass transfer, vol. 45, pp. 703-712, 2009.

[14] A. Bala Jyothi and Vijaya Kumar Varma, S., "Unsteady MHD heat and mass transfer flow past a semi-infinite vertical porous moving plate with variable suction in the presence of heat generation and homogenous chemical reaction," Int. J. of Appl. Math and Mech, vol. 7, no. 7, pp. 20-44, 2011.

[15] Hitesh Kumar, "An analytical solution to the problem of radiative heat and mass transfer over an inclined plate at prescribed heat flux with chemical reaction," $J$. Serb. Chem. Soc., vol. 77, pp. 1-14, 2012. DOI: 03525139/2013/0352-51391200100k

[16] S. Masthanrao, "Chemical reaction effects on mhd free convection flow through a porous medium bounded by an inclined surface," International Journal of Mathematics and Computer Applications Research (IJMCAR), vol. 3, pp. 13-22, 2013. DOI: 10. 18535/ijmcr.

[17] B. D. Coleman and H. Markovitz., "Incompressible second-order fluids," Adv. Appl. Mech., vol. 8, pp. 69101, 1964.

[18] B. D. Coleman and W. Noll., "An applications theorem for functional with application in Continuum Mechanics," Archs Ration Mech. Analysis, vol. 6, pp. 355-360, 1960.

[19] S. K. Nowinski and I. A. Ismail, "Application of multiparameter perturbation methods to elastostatics in development of theoretical and applied mechanics," $N$. A. Shaw, Pergamon Presses, 1965, pp. 11.

[20] R. Choudhury and S. Purkayastha, "An analysis of mixed convective elastic-viscous fluid past a vertical porous plate in presence of induced magnectic field and chemical reaction," Int. J. of Heat and Technology, vol. 32, no. 12, pp. 27-34, 2014.

[21] N. Ahmed and K. Kr. Das, "Chemical reaction effect on mhd free convective mass transfer flow past an impulsively started vertical plate," Int. J. of Heat and Technology, vol. 32, no. 12, pp. 15-22, 2014. 